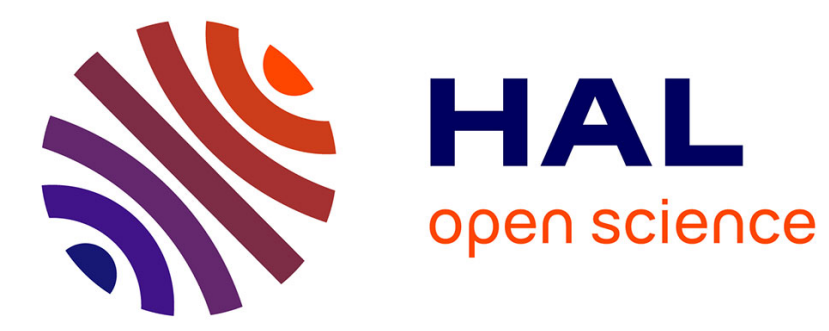

\title{
Résultats du projet ExtraFlo (ANR 2009-2013) sur l'estimation des pluies et crues extrêmes
}

M. Lang, P. Arnaud, Julie Carreau, N. Deaux, L. Dezileau, F. Garavaglia, A. Latapie, L. Neppel, E. Paquet, Benjamin Renard, et al.

\section{- To cite this version:}

M. Lang, P. Arnaud, Julie Carreau, N. Deaux, L. Dezileau, et al.. Résultats du projet ExtraFlo (ANR 2009-2013) sur l'estimation des pluies et crues extrêmes. La Houille Blanche - Revue internationale de l'eau, 2014, 2, pp.5-13. 10.1051/lhb/2014010 . hal-01128341

\section{HAL Id: hal-01128341 \\ https://hal.science/hal-01128341}

Submitted on 9 Mar 2015

HAL is a multi-disciplinary open access archive for the deposit and dissemination of scientific research documents, whether they are published or not. The documents may come from teaching and research institutions in France or abroad, or from public or private research centers.
L'archive ouverte pluridisciplinaire HAL, est destinée au dépôt et à la diffusion de documents scientifiques de niveau recherche, publiés ou non, émanant des établissements d'enseignement et de recherche français ou étrangers, des laboratoires publics ou privés. 


\title{
Résultats du projet ExtraFlo (ANR 2009-2013) sur l'estimation des pluies et crues extrêmes
}

\author{
Michel LANG ${ }^{1}$, Patrick ARNAUD ${ }^{2}$, Julie CARREAU ${ }^{3}$, Nathalie DEAUX ${ }^{4}$, Laurent DEZILEAU ${ }^{5}$, \\ Federico GARAVAGLIA ${ }^{6}$, Audrey LATAPIE ${ }^{1}$, Luc NEPPEL ${ }^{3}$, Emmanuel PAQUET $^{6}$, Benjamin RENARD ${ }^{1}$, \\ Jean-Michel SOUBEYROUX ${ }^{4}$, Benoît TERRIER ${ }^{1}$, Jean-Michel VEYSSEIRE ${ }^{4}$, Yoann AUBERT ${ }^{2}$, \\ Annick AUFFRAY ${ }^{4}$, François BORCHI ${ }^{4}$, Pietro BERNARDARA ${ }^{7}$, Jean-Claude CARRE ${ }^{8}$, \\ Dominique CHAMBON ${ }^{9}$, Thomas CIPRIANI ${ }^{1}$, José-Luis DELGADO ${ }^{10}$, Hilaire DOUMENC ${ }^{9}$, Romain FANTIN ${ }^{4}$, \\ Sylvie JOURDAIN ${ }^{4}$, Krzysztof KOCHANEK ${ }^{1}$, André PAQUIER ${ }^{1}$, Eric SAUQUET ${ }^{1}$, Yves TRAMBLAY ${ }^{3}$ \\ 1. Irstea, Unité de recherche Hydrologie-Hydraulique, 5 rue de la Doua, CS 70077, 69626 Villeurbanne Cedex \\ 2. Irstea, Unité de recherche Ouvrages Hydrauliques et Hydrologie, Route de Cézanne, 13100 Aix-en-Provence \\ 3. HydroSciences Montpellier, cc MSEM, place Eugène Bataillon, 34095 Montpellier Cedex 5 \\ 4. Météo-France, Direction de la climatologie, 42 avenue Gaspard Coriolis, 31057 Toulouse Cedex \\ 5. GéoSciences Montpellier, UMR 5243 - CC 60, Université Montpellier 2, Place E. Bataillon 34095 Montpellier cedex 5 \\ 6. EDF-DTG, 21 avenue de l'Europe, BP 41 Cedex 9, 38040 Grenoble \\ 7. EDF-R\&D LNHE, 6 Quai Watier, 78401 Chatou \\ 8. Artelia Eau et Environnement, 6 rue de Lorraine 38130 Echirolles \\ 9. DREAL Midi-Pyrénées, Service Risques Naturels et Ouvrages Hydrauliques, 1 rue de la Cité administrative, 31074 Toulouse Cedex 9 \\ 10. CETE Méditerranée, Pôle d'activités Les Milles, Avenue Albert Einstein, CS 70499, 13593, Aix-en-Provence Cedex 3
}

\begin{abstract}
RÉSUMÉ. - Cette communication présente les principaux résultats du projet ExtraFlo sur l'estimation des pluies et crues extrêmes. A partir d'un jeu de données conséquent de plusieurs milliers de stations, une procédure d'inter-comparaison a permis d'évaluer les performances d'une trentaine d'implémentations différentes issues de différentes combinaisons (modèle probabiliste, méthode d'estimation, cadre local / régional / mixte local et régional). Le projet ExtraFlo a permis de mettre en évidence le manque de robustesse des approches basées sur le traitement statistique d'une série de quelques dizaines d'années d'observation. Des alternatives intéressantes existent, basées sur l'exploitation des records de pluie et de débit à l'échelle d'une région, de la collecte de données historiques quand cela est possible, de méthodes de simulation basées sur le couplage d'un générateur d'averses avec un modèle hydrologique pour la transformation des pluies en débit, ou des traces morphologiques laissées par les crues anciennes.
\end{abstract}

Mots-clés : pluies extrêmes, crues extrêmes, justesse, robustesse, comparaison de méthodes, distribution prédictive

\section{Main results of a French project on extreme rainfall and flood assessment}

\begin{abstract}
This paper presents a comparison of models for extreme rainfall and flood values. Based on a large set of thousands of rainfall and discharge data, the French ExtraFlo project showed that design estimate of extreme values based on the fitting of a distribution on a limited sample of maximum values is very inaccurate when only a few decades of observation are available. Some interesting alternatives are possible, using a regional approach, or historical data when available, or simulation methods, or sedimentological or geochemical signatures of flood deposits.
\end{abstract}

Key-words: extreme rainfall, extreme flood, reliability, stability, comparison of methods, predictive distribution

\section{INTRODUCTION}

L'estimation du risque hydrologique en un site donné peut être très variable suivant la méthode de prédétermination de pluies ou de débits extrêmes utilisée. Il en résulte pour le non spécialiste une demande forte de clarification, pour connaître les points forts et les lacunes de chaque approche, et voir préciser leur domaine d'application. Il n'est malheureusement pas envisageable de déboucher sur une standardisation complète des méthodes de calcul, comme cela peut exister par exemple pour le génie civil, le domaine d'étude étant trop complexe pour pouvoir être représenté par un seul modèle. L'objectif visé dans le cadre du projet ExtraFlo (ANR 2009-2013) est ici d'entreprendre un travail d'inter-comparaison des approches, pour préciser quelle méthode utiliser en fonction des données disponibles, des particularités hydrologiques du secteur étudié, et du niveau de précision souhaité (fonction des enjeux concernés par le risque inondation). L'originalité est de s'intéresser à un ensemble diversifié de méthodes de prédétermination, en mettant l'accent sur la possibilité de validation à partir de jeux de données de référence.

Nous présentons les principales méthodes d'estimation des pluies et crues extrêmes utilisées en France, puis nous détaillons l'organisation générale du projet avant d'exposer les principales recommandations issues des analyses de comparaison et les pistes de recherche pour les prochaines années. 


\section{LES PRINCIPALES METHODES D'ESTIMATION DES PLUIES ET CRUES EXTRÊMES}

Les études hydrologiques réalisées en France pour la prévention du risque d'inondation ou le dimensionnement d'ouvrages hydrauliques couvrent une large gamme de méthodologies (Lang et Lavabre, 2007). Elles visent à déterminer des valeurs de référence de pluie ou de crue extrême, associées à une période de retour $T$ donnée ou à un maximum connu. Suivant la nature des enjeux, la cible d'étude sera relative à :

- une probabilité annuelle de dépassement $p=1 / T$, qui va de $10^{-1}$ (réseau routier secondaire, réseau d'assainissement), $10^{-2}$ (autoroute, zone habitée), $10^{-3}$ (aléa majeur pour la Directive Inondations, barrage rigide) jusqu'à $10^{-4}$ (barrage meuble, centrale nucléaire) ;

- la crue historique la plus forte connue ou la crue centennale si celle-ci est plus forte (PPR inondation) ;

- la limite du lit majeur susceptible de contenir l'enveloppe des plus grandes crues passées (étude hydro-géomorphologique).

On peut distinguer trois familles de méthodes de prédétermination des pluies et des débits.

\section{II.1. Théorie des valeurs extrêmes}

La théorie des valeurs extrêmes (Embrechts et al., 1997, Coles, 2001, Beirlant et al., 2004), fondée sur des résultats de la théorie des probabilités, offre un cadre mathématique rigoureux pour l'estimation des probabilités de valeurs rares à extrêmes de pluie et de débit. La loi GEV (loi généralisée des extrêmes, Generalized Extreme Value distribution) (Fisher et Tippet, 1928, Jenkinson, 1955) et la loi GP (loi de Pareto généralisée, Generalized Pareto distribution) (Pickands, 1975) permettent respectivement de caractériser le comportement en loi de maxima et de dépassements de seuils élevés. Une hypothèse forte de l'application directe qui est faite de cette théorie réside dans l'écart entre le comportement idéal et asymptotique d'une loi mathématique et le comportement réel d'un phénomène physique observé dans des gammes de fréquence, nécessairement éloignées de l'asymptote. De plus l'homogénéité des évènements n'est pas vérifiée et les processus - atmosphériques, hydrologiques et hydrauliques - peuvent être perturbés par des seuils physiques.

\section{II.1.1. Analyse locale}

L'application de la théorie des valeurs extrêmes sur une série courte de quelques dizaines d'années conduit à de fortes incertitudes d'estimation des événements extrêmes, liées à la distribution d'échantillonnage (forte sensibilité à la présence de valeurs singulières), à la possible hétérogénéité des populations fondant l'ajustement, et au choix du modèle probabiliste. Or les événements courants ne sont que peu informatifs, au sens statistique, pour la prédétermination des valeurs fortes de pluie ou de débit. La genèse physique de ces valeurs extrêmes est en effet bien différente de celle des événements courants. Appliquées sur de longues séries, ces approches redeviennent pertinentes pour l'estimation des valeurs extrêmes, d'où la préoccupation des hydrologues de développer des méthodes permettant d'incorporer des informations complémentaires aux seules séries locales de données de quelques dizaines d'années.

\section{II.1.2. Analyse régionale}

Une première façon d'augmenter la taille de l'échantillon d'analyse consiste à élargir le domaine spatial d'observation et à analyser simultanément les observations de différents postes de mesure d'une zone supposée homogène. La première application (Dalrymple, 1960) considérait que les distributions des sites d'une même région homogène étaient identiques, à un facteur multiplicatif près, appelé indice de crue. De nombreux raffinements ont été apportés depuis (Hosking et Wallis, 1997a, St-Hilaire et al., 2003, Onibon et al., 2004, Ribatet et al., 2006, 2007), notamment sur la notion d'homogénéité hydrologique (découpage géographique, puis voisinage hydrologique) et sur le mode de traitement statistique qui permet de combiner information locale et régionale, avec un poids fonction du degré d'homogénéité-hétérogénéité du jeu régional de données. Une des limitations de ces approches est souvent de ne pas considérer l'impact de la dépendance spatiale entre données, ce qui peut conduire à sous-estimer l'incertitude finale sur l'estimation des quantiles de pluie et de débit de référence (Hosking et Wallis, 1997b). Ces méthodes de régionalisation peuvent par ailleurs être utilisées pour estimer les quantiles de pluie ou de débit en site non jaugé, en combinant une loi régionale adimensionnelle (issue du formalisme précédent) et une méthode d'estimation de l'indice de pluie ou débit (régression multiple à partir de paramètres climatiques ou physiographiques explicatifs).

\section{II.1.3. Intégration de données historiques}

Une seconde approche consiste à réunir des informations sur les crues anciennes à partir de sources documentaires. L'intérêt est évidemment d'élargir l'échelle temporelle d'analyse, en utilisant des événements exceptionnels, qui sont potentiellement les plus riches d'information pour l'estimation des quantiles extrêmes. Le cadre statistique d'analyse doit être adapté (Miquel, 1984 ; Parent et Bernier, 2003 ; Neppel et al., 2010 ; Gaume et al., 2010 ; Payrastre et al., 2011) au fait que l'on ne dispose que de données incomplètes, en général principalement sur les crues ayant eu de forts impacts, et que la reconstitution du débit de pointe des crues historiques est entachée d'incertitudes beaucoup plus importantes qu'avec des données récentes (cf. transformation hauteur-débit). Une autre incertitude est liée à l'estimation du seuil de perception des crues historiques, fixé de façon empirique et susceptible d'évoluer dans le temps.

\section{II.2. Connaissance des processus de transformation pluie-débit}

\section{II.2.1. Transformation d'un hyétogramme de projet en hydrogramme de projet}

Cette approche consiste à transformer une pluie de référence en crue de référence, en utilisant un modèle hydrologique de transformation de la pluie en débit, adapté aux caractéristiques physiques du bassin versant. La pluie est représentée par un hyétogramme de projet. Le fonctionnement du bassin versant est déterminé par des paramètres synthétiques qui décrivent les processus d'infiltration, de ruissellement et éventuellement d'évaporation, d'écoulement souterrain et de fonte des neiges.

Un modèle hydrologique fréquemment utilisé dans le monde est l'hydrogramme unitaire du Soil Conservation Service (Ven Te Chow, 1964), qui décrit le fonctionnement du bassin versant par deux paramètres synthétiques : le temps de concentration et le coefficient $C N$ ( « curve number »), associés respectivement à la cinétique de ruissellement et à la capacité d'infiltration. Il existe de nombreux autres modèles déterministes, parmi lesquels GR4J (Perrin, 2000), 
MORDOR (Garcon, 1996 ; Paquet, 2004), Topmodel (Beven et Kirby, 1979) ... Certains modèles hydrologiques ont été développés et calés sur une typologie particulière de bassins versants et d'événements, et ne sont donc pas nécessairement bien adaptés pour décrire toutes les situations. Il est important que le modèle hydrologique soit bien calé sur des événements significatifs de crue, pour calibrer correctement les paramètres.

L'intérêt de cette approche est de pouvoir spécifier dans le détail la forme de la pluie de projet et de décrire le processus de rétention en eau du bassin par un modèle hydrologique. La difficulté réside dans le choix adapté des conditions initiales de saturation du bassin qui permette d'attribuer une période de retour à la crue de référence. Une hypothèse forte de ces approches est la correspondance directe entre une pluie de projet de période de retour donnée avec une crue de projet de même période de retour.

\section{II.2.2. Modèles probabilistes simplifiés pluie-débit}

La méthode du Gradex, développée par EDF (Guillot et Duband, 1967) pour la sécurité des grands barrages, a beaucoup été utilisée en France depuis plus de 30 ans pour l'estimation des débits de fréquences rare et exceptionnelle (période de retour 1000 à 10000 ans). La méthode utilise l'information pluviométrique pour extrapoler la distribution de fréquence des débits. Son succès est en particulier lié à sa (relative) facilité de mise en oeuvre qui résulte de la simplification extrême du processus de transformation de la pluie en débit. La méthode Speed (Cayla, 1993) donne directement la distribution de la pointe de crue, à partir de la distribution des pluies journalières et d'une estimation de la capacité maximale d'infiltration du bassin versant (en mm). Le modèle Agregee (Margoum, 1992 ; Margoum et al., 1994) assouplit les hypothèses de base de la méthode $\mathrm{du}$ Gradex, en autorisant pour la pluie des lois non strictement à décroissance exponentielle et en introduisant une fonction analytique pour raccorder progressivement le domaine des crues observées et celui des crues exceptionnelles (saturation progressive du bassin versant).

\section{II.2.3. Couplage d'un simulateur d'averses et d'un modèle hydrologique}

Une approche plus complète consiste à utiliser un générateur d'averses pour mieux apprécier l'aléa pluviométrique et un modèle pluie-débit pour mieux représenter la transformation pluie-débit. Le modèle Shypre (Cernesson et al., 1996 ; Lavabre et al., 1998 ; Arnaud et Lavabre, 2000 et 2002), acronyme de Simulation d'HYdrogrammes pour la PREdétermination des crues, permet de générer de très longues chroniques de pluies horaires (sur 100000 ans, par exemple) à partir d'hypothèses sur la structuration des averses et les lois de probabilités sous-jacentes. On déduit ensuite les hydrogrammes de crue par application d'une modélisation de la pluie en débit. Le classement des pluies et des débits simulés permet d'en extraire les valeurs maximales, de tracer les distributions de fréquence " empirique » des pluies et des débits de toutes durées, et d'en déduire les quantiles pour une large gamme de périodes de retour, de 2 à 1000 ans, sans supposer à ce stade une loi de probabilité particulière. Une version régionale de Shypre, dénommée Shyreg (Arnaud et Lavabre, 2012, 2014), permet d'estimer les quantiles de pluie et de débit en tout point du territoire français.

La méthode SCHADEX (Paquet et al., 2006, 2013) estime l'aléa de crue en composant les aléas pluie et état hydrique du bassin. Pour une saison donnée, les pluies sont probabilisées au moyen de lois exponentielles calées sur les sous-populations correspondant à huit types de temps, recombinées avec la probabilité relative d'occurrence de ces types de temps (distribution MEWP, Garavaglia et al., 2010, 2011). Une méthode simplifiée de génération d'averses permet de croiser quasi exhaustivement les aléas précipitations et état hydrique du bassin en exploitant une chronique climatologique continue enregistrée sur le bassin. La réponse de celui-ci aux pluies simulées est obtenue à l'aide d'un modèle hydrologique (modèle MORDOR pluie-température-débit, prenant en compte la composante nivale), et conduit à la probabilisation des écoulements simulés et la détermination de la distribution des débits de crue.

\section{II.3. Exploitation de données naturalistes sur les crues}

\section{II.3.1. Hydro-géomorphologie}

L'approche hydrogéomorphologique (Garry et al., 1996) permet, à partir d'une analyse des cartes topographiques au $1 / 25000$, de stéréographies aériennes et de reconnaissances de terrain par un géomorphologue, de distinguer les différentes unités du lit d'écoulement de la rivière : le lit mineur correspond à la section usuelle d'écoulement, le lit moyen assure la transition entre le lit mineur et le lit majeur, et le lit majeur, qui fonctionne pour les crues importantes, correspond à la plaine alluviale. Les limites de ce dernier lit donnent une idée de l'extension maximale du champ d'inondation. Cette méthode a fait l'objet d'une application généralisée à l'ensemble de la région Midi-Pyrénées (Prunet, 1998 ; Vidal, 2000 ; Lambert et al., 2001) et des principaux cours d'eau des régions Languedoc-Roussillon et Provence- Alpes-Côte-d'Azur (Delgado, 2006), dans le cadre de la mise en place d'une cartographie informative sur les zones inondables.

\section{II.3.2. Paléo-hydrologie}

Une approche complémentaire, appelée paléo-hydrologie, permet par ailleurs la reconstitution du niveau atteint par les plus fortes crues depuis plusieurs millénaires, en s'intéressant aux dépôts laissés par les crues, soit directement dans le champ d'inondation, soit dans des cavités surplombant le lit mineur. Des techniques d'analyse stratigraphique et de datation des dépôts (C14, thermoluminescence) sont alors utilisées pour positionner en altitude les plus forts événements, et proposer une chronologie de crues (House et al., 2002 ; Benito et al., 2004). Les résultats obtenus sur le bassin de l'Ardèche (Sheffer et al., 2003a) ont montré que la crue de 1890, la plus forte connue sur une période historique de quatre siècles, n'avait pas été dépassée depuis plusieurs milliers d'années. Inversement, les analyses paléohydrologiques menées sur deux grottes situées dans les gorges du Gard (Sheffer et al., 2003b) ont mis en évidence la trace de cinq crues ayant dépassé le niveau de la crue de septembre 2002, dont trois sur la période 1400-1800. Les données historiques sur les crues des derniers siècles peuvent servir pour contrôler les résultats des datations de crues récentes, et inversement les données paléohydrologiques peuvent élargir le cadre temporel issu d'une recherche dans les sources d'archives.

Les approches naturalistes sont intéressantes dans la mesure où elles donnent des éléments objectifs sur les crues majeures survenues sur le bassin versant. Elles soulèvent toutefois des difficultés d'interprétation pour déterminer si les niveaux de crues atteints dans les conditions climatiques et géomorphologiques du passé restent représentatifs 
$\mathrm{du}$ risque d'inondation futur (cf. variations climatiques à l'échelle pluriséculaire, divagation latérale du cours d'eau dans la plaine d'inondation, changement de l'occupation du sol sur le bassin).

\section{ORGANISATION GENERALE DU PROJET EXTRAFLO}

\section{III.1. Constitution de jeux de données test}

La première partie du projet a été consacrée à la constitution de jeux de données test :

- Données pluviométriques sur la moitié sud de la France (diagonale au sud d'une ligne Bayonne-Strasbourg), qui couvre plusieurs régimes climatiques (océanique, méditerranéen et montagnes du Sud de la France), avec une centaine de longues séries quotidiennes, un jeu régional sur le secteur Méditerranée-Alpes du Nord (1900 postes quotidiens ; 230 postes avec données infra-journalières), un jeu régional à pas de temps fin $(6 \mathrm{mn})$ sur le Grand Lyon (28 postes depuis 1993) ; et des actions de digitalisation de séries à pas de temps fin (200 années-stations).

- Données hydrométriques sur l'ensemble de la métropole : 150 séries quotidiennes d'au moins 40 années, échantillons de valeurs maximales issus de 1400 séries quotidiennes, 12 longues séries avec données historiques sur deux à trois siècles.

- Données naturalistes sur le Gard : prospection générale et sélection d'une vingtaine de sites dans les gorges, avec prélèvements d'échantillons de sédiments de crue pour datation ; collecte des données cartographiques hydromorphologiques disponibles, recensement des données topographiques et hydrologiques disponibles en vue d'une modélisation hydraulique ; échanges avec la DREAL Midi-Pyrénées pour le choix de sites d'étude sur le bassin de la Garonne.

- Sélection de 24 crues remarquables et documentation à titre d'exemple de 4 épisodes (13 février 1990, 10-13 juin 2000, 3 mai 2007, 15 juin 2010) : analyse météorologique, champs de pluie, processus hydrologiques, hydrogrammes remarquables, références bibliographiques.

\section{III.2. Mise au point d'une stratégie commune d'inter-comparaison et de validation}

L'état de l'art précédent montre la grande diversité de méthodes de prédétermination des pluies et crues extrêmes, à laquelle vient se rajouter un foisonnement de variantes à l'intérieur de chaque famille. Par la suite, pour éviter toute ambigüité nous utiliserons le terme «implémentation » pour distinguer une variante particulière. Par nature, l'évaluation de méthodes dédiées aux valeurs extrêmes est délicate, puisque l'on s'intéresse à des événements en général rarement observés voire jamais atteints localement. La théorie des valeurs extrêmes repose sur un cadre mathématique rigoureux et il existe de nombreux résultats théoriques ou obtenus par simulation pour apprécier et comparer la pertinence de telle distribution (Fréchet, Gumbel, Weibull) ou de telle méthode d'estimation (maximum de vraisemblance, moments, moments pondérés). Mais ces résultats ne sont valables que d'un point de vue asymptotique, pour des tailles d'échantillon bien plus larges que celles rencontrées en hydrologie. De plus, la théorie des valeurs extrêmes est basée sur l'hypothèse que les valeurs de l'échantillon sont indépendantes et identiquement distribuées, ce qui n'est pas la règle en hydrologie.
Il est possible de mettre en place des tests statistiques pour apprécier si la distribution théorique issue d'une méthode-implémentation donnée est cohérente ou pas avec les observations. Toutefois la puissance de ces tests reste faible pour les valeurs extrêmes, lorsque la période de retour cible est bien plus importante que la taille de l'échantillon analysé. Deux types d'évaluations peuvent être distingués :

- Le premier type s'applique à toutes les méthodes pouvant faire l'objet d'une application automatisée. Elles ont l'avantage de produire des estimations répétitives et objectives, sur de vastes jeux de données, ouvrant la voie à une qualification statistique rigoureuse.

- Le deuxième type s'applique à des méthodes requérant de l'expertise humaine, ne pouvant être appliquées automatiquement sur de grands jeux de données. L'analyse s'appuie alors sur des applications détaillées à des cas tests emblématiques et bien documentés, et examine finement les processus hydrologiques et statistiques en jeu dans l'extrapolation.

\section{III.3. Inter-comparaison de méthodes statistiques sur un large jeu de données}

Les jeux de données utilisés ont été constitués sur des critères de qualité de mesure, de durée minimale d'observation et d'absence d'influence anthropique :

- Données pluviométriques : 1122 stations x 35 années (nombre moyen par station) $=39270$ années-stations ;

- Données hydrométriques : 1170 stations x 40 années (nombre moyen par station) $=46800$ années-stations.

Avec un tel effectif, il devient possible de détecter pour une implémentation donnée si l'estimation qui est faite par exemple de la pluie centennale est effectivement dépassée en moyenne une fois sur 100 ans, ou au contraire "trop » ou «pas assez » souvent. Il faut évidemment raisonner en régions homogènes, et mettre au point des procédures permettant de «mélanger » les données à l'échelle d'un jeu de plusieurs centaines de stations. Le dépassement d'un seuil d'intensité pluviométrique de $100 \mathrm{~mm} / \mathrm{jour}$ n'a pas la même signification suivant le régime climatologique, de même qu'une crue de $1000 \mathrm{~m}^{3} / \mathrm{s}$ peut être tout à fait courante sur un grand bassin et exceptionnelle voire impossible sur un petit bassin versant.

Le principe général retenu pour l'inter-comparaison des méthodes a consisté à diviser le jeu de données en jeu de calage sur lequel les paramètres des différents modèles-implémentations sont calés, puis à évaluer les performances des méthodes sur un jeu de validation. Deux familles de critères ont été retenues, relatives à la justesse et à la stabilité ou robustesse. La justesse renseigne sur la capacité d'une implémentation à délivrer des estimations cohérentes avec les observations ; la stabilité sur la sensibilité des estimations à l'échantillon disponible. Il est ainsi possible de mettre en évidence les méthodes qui sur ou sous-estiment de façon systématique les quantiles, et ensuite de départager parmi les méthodes « justes », c'est à dire avec un biais faible, celles qui sont les plus robustes. Une méthode hyper-paramétrée, dont les résultats sont jugés tout à fait convenables sur l'échantillon de calage, va être détectée comme non robuste sur l'échantillon de validation.

Pour les précipitations extrêmes, nous avons testé : 1/les approches basées sur la théorie des valeurs extrêmes : lois GEV-Gumbel ou GP-Expo (à l'échelle locale, régionale, locale-régionale), distribution MEWP par type de temps ; 2/ l'approche SHYREG-pluie par simulation d'averses (à l'échelle locale, régionale, locale-régionale). A noter que 
ce protocole ne traite que des pluies locales, et que les pluies spatiales (à l'échelle du bassin versant) et leurs méthodes associées (fonction d'abattement notamment) n'ont pas été évaluées dans le projet. Pour les crues extrêmes, nous avons testé : 1/ les approches basées sur la théorie des valeurs extrêmes : lois GEV-Gumbel (à l'échelle locale, régionale, locale-régionale), lois LogNormale, Pearson III et Log Pearson III ; 2/l'approche SHYREG-débit par simulation d'averses couplée à un modèle hydrologique (à l'échelle locale, régionale, locale-régionale).

\section{III.4. Comparaison de méthodes d'estimation sur quelques bassins test}

D'autres méthodes-implémentations ne pouvaient facilement être testées à si grande échelle sur plusieurs centaines de stations, du fait de leur complexité, du temps nécessaire ou disponible pour réaliser une expertise au cas par cas, de la difficulté à disposer des données nécessaires ... Il s'agit pour les crues extrêmes :

- des approches basées sur la théorie des valeurs extrêmes par analyse des crues historiques et de l'approche multifractales ;

- des approches basées sur l'information pluviométrique et une transformation simplifiée pluie-débit : méthodes du Gradex, Agregee, Speed ;

- l'approche SCHADEX par simulation de pluies couplée à un modèle hydrologique ;

- l'approche SHYREG-débit dans une application expertisée ; - des approches naturalistes par étude hydro-géomorphologique ou paléo-hydrologique.

Nous avons ainsi réalisé une action de comparaison sur un nombre limité de bassins versants, sur l'Ardèche, le Gard, le Tech pour les méthodes probabilistes, et sur le Gard et la Garonne pour les approches naturalistes. Il n'est alors pas possible de conclure sur la justesse de ces approches, mais a minima de les positionner les unes par rapport aux autres, en termes de valeur centrale et de dispersion, et de voir les complémentarités entre cartographie hydro-géomorphologique et modélisation hydrologique et hydraulique.

\section{RECOMMANDATIONS POUR L'ESTIMATION DES PLUIES ET CRUES EXTREMES}

\section{IV.1. Pluies extrêmes sur site jaugé}

Les analyses menées dans le projet ExtraFlo ont montré que l'approche standard d'estimation des pluies extrêmes par ajustement des paramètres d'une loi GEV (max-annuel) ou GP (sup-seuil) à partir d'une série locale n'est pas recommandée : si l'on conserve trois paramètres, on ne dispose pas assez d'information pour caler correctement le paramètre de forme de la distribution. L'estimation est peu robuste, avec une forte sensibilité à la présence de valeurs singulières dans l'échantillon (c.a.d bien différentes du reste de la distribution). Si l'on utilise une loi à décroissance exponentielle (loi de Gumbel ou loi exponentielle) sur un échantillon global de précipitations journalières ou infra-journalières sans découpage préalable en sous-populations homogènes, on sous-estime la distribution des pluies fortes.

Trois méthodes peuvent être recommandées :

- Estimation par une approche régionale de type index-flood avec une loi GEV. Cette méthode a donné les meilleurs résultats en termes de justesse et de robustesse. Elle est simple d'application (une fois développée), avec d'une part une distribution régionale calée à partir des séries appartenant à la région d'étude, et d'autre part un index de pluie estimé à partir de la série locale. On dispose par ailleurs d'une estimation des intervalles de confiance. Elle nécessite par contre un développement spécifique pour chaque durée de pluie étudiée (pluie maximale journalière, horaire ...).

- Estimation par le modèle de simulation SHYREGPluies. Cette méthode a donné des résultats très proches en termes de justesse et de robustesse. Elle fournit une estimation pour une pluie locale à l'échelle d'un pixel de $1 \mathrm{~km}^{2}$. L'intérêt de la méthode est qu'elle fournit directement une estimation des quantiles de pluie, pour des cumuls entre $1 \mathrm{~h}$ et $72 \mathrm{~h}$.

- Estimation par le modèle par type de temps MEWP (Multi-Exponential Weather Pattern). Ce type de distribution a donné de bons résultats en terme de robustesse, et en second rang pour la justesse, bien qu'ajusté à partir d'une série locale de précipitations. L'information sur les types de temps s'avère un moyen intéressant d'améliorer la qualité des estimations. La méthode est directement applicable pour une pluie de bassin. L'hypothèse exponentielle serait cependant à reconsidérer pour certains types de temps, dans certaines régions du pourtour méditerranéen, si l'on s'en tient à la classification actuelle, pour remédier à une sous-estimation des quantiles des pluies locales.

\section{IV.2. Pluies extrêmes sur site non jaugé}

Bien que très documentée dans la littérature, l'approche consistant à estimer les paramètres d'une loi GEV au droit de tous les postes voisins et à établir ensuite une régression expliquant ces paramètres en fonction de co-variables géographiques ne donne pas de bons résultats. Il est préférable d'utiliser :

- Estimation par le modèle de simulation SHYREGPluies. Cette méthode a donné les meilleurs résultats en termes de justesse et de robustesse. Une estimation des quantiles est disponible sur l'ensemble du territoire métropolitain, pour des cumuls de pluies entre $1 \mathrm{~h}$ et $72 \mathrm{~h}$, et des périodes de retour de 2 à 1000 ans.

- Estimation par une approche régionale de type index-flood avec une loi GEV. Cette méthode a donné de bons résultats en terme de justesse, et un peu moins robustes qu'avec le modèle SHYREG-Pluies.

La comparaison entre modèle local-régional et purement régional ne montre qu'une dégradation légère des performances en n'exploitant aucune information locale (site non jaugé). Ce constat plutôt rassurant vient de la relative bonne adéquation du réseau de mesure pluviométrique journalier à la variabilité spatiale des précipitations. Il est à nuancer pour les intensités de précipitation infra-journalière ou en zone d'altitude, avec une densité de réseau bien inférieure.

\section{IV.3. Crues extrêmes sur site jaugé}

La confiance que l'on peut accorder dans l'extrapolation d'une distribution est plus faible pour les crues que pour les pluies extrêmes. Au-delà de la période de retour 50 ans, on observe une forte variabilité de comportement asymptotique, suivant la diversité de régime hydrologique (crue pluviale, nivale, mixte) et de bassin versant (capacité de rétention, superficie). 
Ceci a été bien illustré lors de la comparaison de l'ensemble des méthodes sur quelques bassins tests : l'approche standard par ajustement d'une distribution sur les maximum de crue extrapole dans la continuité des observations, et peut conduire à de forts biais d'estimation en comparaison avec la distribution empirique des crues historiques disponibles sur une plus longue période. La figure suivante sur le Gardon à Corbès montre que les approches locales (loi Gumbel et GEV), qui n'exploitent que la série locale (1970-2012), donnent une estimation de la crue centennale un peu inférieure à $1000 \mathrm{~m}^{3} / \mathrm{s}$, alors que l'approche GEV régionale dépasse un peu $1000 \mathrm{~m}^{3} / \mathrm{s}$, et que les méthodes basées sur l'information pluviométrique (SHYREG, SCHADEX, GRADEX, AGREGEE, SPEED) sont bien au-dessus, entre 1300 et $1600 \mathrm{~m}^{3} / \mathrm{s}$. L'exploitation de l'information historique, avec trois crues supérieures à $1200 \mathrm{~m}^{3} / \mathrm{s}$ sur la période 1744-1970 est cohérente avec le dernier groupe de méthodes basées sur les pluies.

Comme pour les pluies, l'approche standard par ajustement des paramètres d'une distribution à partir d'une série locale n'est pas recommandée pour l'estimation des crues extrêmes. Il est important d'exploiter des informations complémentaires pour conforter l'extrapolation de la distribution des crues. Nous recommandons d'utiliser :

- Les méthodes de simulation basées sur l'information pluviométrique. La méthode SHYREG-débit a été testée sur un jeu étendu de données hydrométriques (519 stations en mode local, 1076 stations en mode régional). Parmi les méthodes applicables en mode semi-automatique, elle a donné les meilleurs résultats en termes de justesse et de robustesse. Une tendance à la sur-estimation a toutefois été détectée sur le secteur méditerranéen, estimée autour de 5 à $10 \%$ en moyenne. La méthode SCHADEX relève de la même famille de méthodes. Dans son application industrielle, elle s'appuie sur l'expertise hydrologique du chargé d'étude. Elle n'a pas donc pu faire l'objet d'une application semi-automatique sur le jeu de données hydrométriques du projet, et donc n'a pas pu être évaluée selon le protocole décrit en section III.3. Cependant, son application sur cinq bassins tests en secteur méditerranéen a montré que l'extrapolation vers les valeurs extrêmes qu'elle propose est dans la même famille que celles des évaluations GRADEX et SHYREG-débit. Une étude basée sur 60 études industrielles expertisées d'EDF et publiée en 2012 aboutit à un constat similaire sur l'estimation des valeurs extrêmes par SCHADEX et GRADEX (Paquet et al., 2012).

- L'approche locale-régionale de l'index-flood avec une loi GEV. La méthode a donné de bons résultats, assez proches de ceux de SHYREG-débit, et de meilleure qualité en secteur méditerranéen. Lors de la comparaison sur bassins tests en secteur méditerranéen, cette approche a donné des estimations légèrement inférieures à celles obtenues à partir d'une information pluviométrique. En secteur océanique, l'utilisation d'une loi de Gumbel semble acceptable avec une approche locale-régionale. Insistons ici sur l'importance des données locales : une approche purement régionale donne des résultats très médiocres.

- L'information historique sur les crues. La méthode n'a pas été testée à grande échelle (cf. nombre limité de bassins où une information de ce type est immédiatement accessible), mais elle a donné sur quelques bassins tests des résultats voisins de ceux obtenus à partir d'une information pluviométrique.

L'obtention d'hydrogrammes de crue de référence passe généralement par la détermination du débit de pointe $Q p(T)$ relatif à une période de retour $T$ cible et par l'utilisation d'une forme moyenne de crue. Un contrôle de cohérence peut être fait sur le volume de la crue, à partir de quantiles de crue $Q d(T)$ pour un débit moyen maximal calculé sur différentes durées $d$. Les méthodes par simulation SHYREG et SCHADEX offrent une alternative intéressante à l'hypothèse d'une forme moyenne de crue, avec l'introduction d'une variabilité des formes de crue. Une application a été réalisée sur une retenue virtuelle localisée sur le bassin du Tech pour la simulation de cotes extrêmes dans une retenue (Paquet et al., 2014).

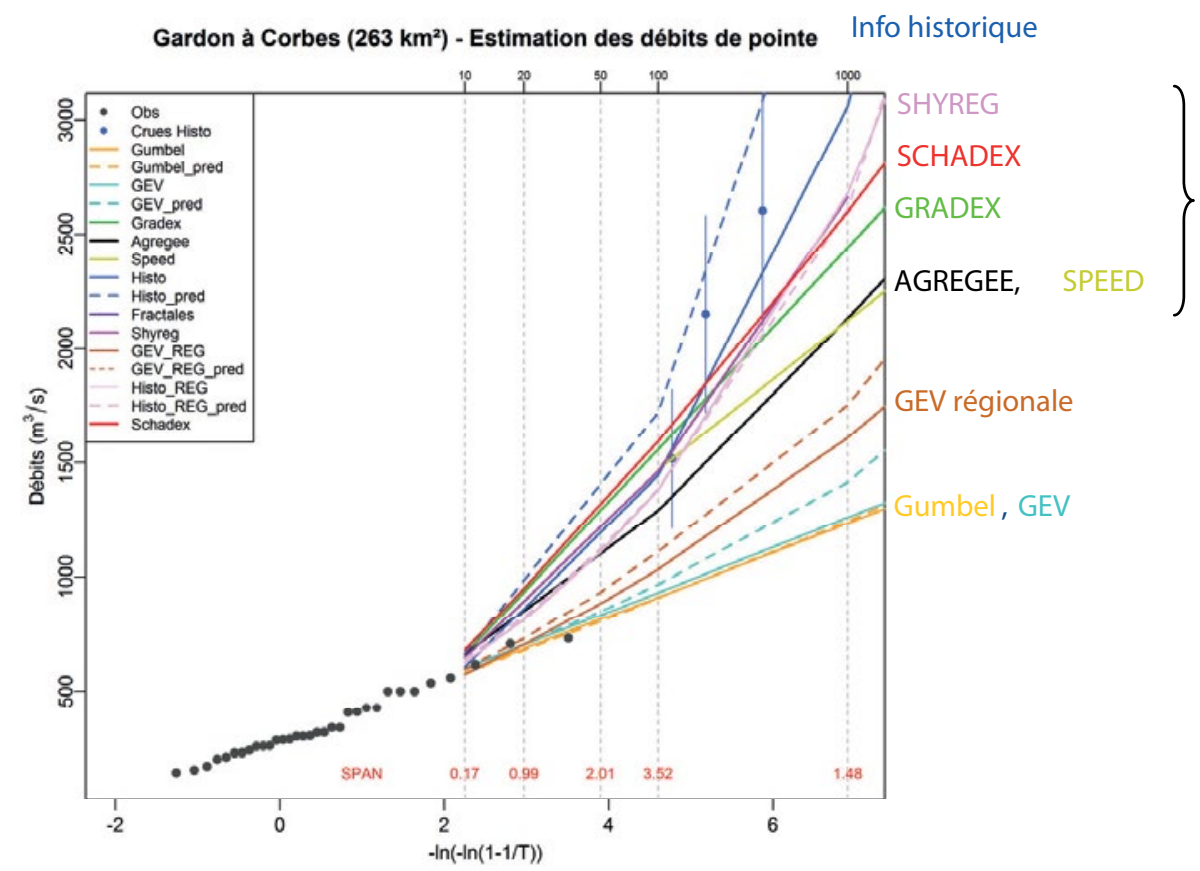

Figure 1 : Comparaison des quantiles de crue en débit de pointe sur le Gardon à Corbès. 


\section{IV.4. Crues extrêmes sur site non jaugé}

La qualité des estimations est bien dégradée lorsque l'on ne dispose pas de mesure hydrométrique au droit du site cible. L'interpolation spatiale est plus difficile à réaliser que pour les précipitations extrêmes, car le réseau hydrographique introduit des discontinuités bien plus fortes. En l'état toutes les méthodes testées ont une justesse qui reste assez médiocre. Comme pour les précipitations, l'approche consistant à estimer les paramètres d'une loi GEV au droit de tous les postes voisins et à établir ensuite une régression expliquant ces paramètres en fonction de co-variables géographiques ne donne pas de bons résultats. Il est préférable d'utiliser :

- Le modèle de simulation SHYREG-Débit. Cette méthode est de loin la plus robuste, et un peu plus juste que les autres. Une tendance à la sur-estimation des quantiles a été détectée en secteur méditerranéen. Les quantiles de crue sont disponibles sur l'ensemble du territoire métropolitain, pour le débit de pointe $Q_{p}$ et les débits moyens $Q d$ ( $d$ entre $1 \mathrm{~h}$ et $72 \mathrm{~h}$ ), et des périodes de retour de 2 à 1000 ans. Un certain nombre de limitations sont rappelées aux usagers (Arnaud et al., 2013) : l'approche n'a pas été validée hors de la gamme usuelle de superficie des bassins jaugés $\left[10 ; 2000 \mathrm{~km}^{2}\right]$ et le cas de bassins à fonctionnement spécifique (influence nivale, karst, barrages, zone urbaine) mérite un complément d'étude. - L'approche régionale de type index-flood. Cette méthode a donné des résultats corrects, un peu moins robustes qu'avec le modèle SHYREG-Pluies. Les résultats sont meilleurs en secteur méditerranéen avec une loi GEV régionale.

\section{IV.5. Emprise de la zone inondée pour une crue extrême}

La comparaison sur la Garonne et sur le Gardon des différents lits définis par approche géomorphologique et des zones inondées calculées avec les modèles hydrauliques montre une continuité dans les valeurs de débits de remplissage sur des linéaires relativement homogènes (Latapie et al., 2014). Des différences ont été relevées en secteur anthropisé où la forme du lit n'est plus directement interprétable. Le choix d'une approche (modélisation hydraulique ou géomorphologie) dépend principalement des données disponibles (topographie de la rivière, fonctionnement des ouvrages, hydrologie) et du temps imparti a l'étude. En fonction des particularités des différents tronçons identifiés, les avantages et les limites des différentes approches sont détaillés dans le tableau suivant.

En zone urbaine, l'approche géomorphologique donne simplement les limites "naturelles" de l'encaissant : la modélisation hydraulique est indispensable pour apprécier correctement l'impact des digues éventuelles et les conditions d'écoulement dans les rues. La présence d'îles ne pose pas de problème particulier en géomorphologie, alors qu'il est nécessaire de disposer de levés détaillés de la topographie pour alimenter les modèles hydrauliques. En zone de confluence, l'approche géomorphologique donne directement l'enveloppe des zones inondées par la crue soit du cours d'eau principal soit de l'affluent, sans information spécifique sur l'origine de la crue. L'approche hydraulique nécessite une hypothèse sur le risque de crues simultanées sur les deux branches et l'emploi d'une modélisation 2D lorsque des recirculations existent. En zone rurale, l'interprétation géomorphologique de l'encaissant est délicate lorsque celui-ci est peu marqué, alors que le modèle hydraulique donne correctement l'extension des zones inondées.

La modélisation hydraulique 1D est prise en défaut lorsque les écoulements sont complexes : fonctionnement en casiers multiples, recirculations latérales, ligne d'eau non horizontale sur un profil en travers situé en courbe. Dans ce type de configuration, la modélisation hydraulique 2D permet de mieux simuler les écoulements, sous réserve de disposer d'une topographie bien détaillée. Des progrès récents ont été obtenus sur la topographie en lit majeur via les campagnes Lidar. Pour la topographie du lit mineur, des levés de terrain restent nécessaires et peuvent représenter un coût d'acquisition non négligeable.

A noter que pour les deux approches, il est très intéressant d'exploiter l'information sur les crues historiques : elles permettent de recouper les limites obtenues par l'approche géomorphologique, et d'aider au calage du modèle hydraulique quand des laisses de crue sont disponibles. Par ailleurs, les deux approches sont complémentaires : la géomorphologie peut apporter des informations utiles à un modèle hydraulique, notamment lors de la constitution de la topologie du modèle et de ses différents chenaux d'écoulement. L'hydraulique peut permettre de recouper des anomalies dans les limites de l'approche géomorphologique (discontinuités dans le débit de l'encaissant) et également d'interpoler en secteur aménagé.

\section{CONCLUSIONS ET PISTES DE RECHERCHE}

Le projet ExtraFlo a permis de mettre en évidence le manque de robustesse des approches basées sur le traitement statistique d'une série de quelques dizaines d'années d'observation. Des alternatives intéressantes existent, basées sur l'exploitation des records de pluie et de débit à l'échelle d'une région, de la collecte de données historiques quand cela est possible, de méthodes de simulation basées sur le couplage d'un générateur d'averses avec un modèle

\begin{tabular}{|c|c|c|c|c|c|c|}
\hline \multirow[t]{2}{*}{ Secteur } & \multicolumn{2}{|c|}{ Géomorphologie } & \multicolumn{2}{|c|}{ Modèle hydraulique 1D } & \multicolumn{2}{|c|}{ Modèle hydraulique 2D } \\
\hline & + & - & + & - & + & - \\
\hline $\begin{array}{l}\text { Zone urbaine } \\
\text { endiguée }\end{array}$ & \multirow{4}{*}{$\begin{array}{l}\text { Rapide à mettre } \\
\text { en œuvre } \\
\text { et coût limité }\end{array}$} & $\begin{array}{l}\text { Impact ouvrage } \\
\text { non pris en compte }\end{array}$ & \multirow{4}{*}{$\begin{array}{l}\text { Quantification } \\
\text { des phénomènes } \\
\text { et rapidité } \\
\text { du calcul }\end{array}$} & $\begin{array}{l}\text { Topographie } \\
\text { du lit majeur }\end{array}$ & \multirow{4}{*}{$\begin{array}{l}\text { Accès au champ } \\
\text { des vitesses } \\
\text { et adapté } \\
\text { aux écoulements } \\
\text { complexes }\end{array}$} & $\begin{array}{l}\text { Détails } \\
\text { ouvrages }\end{array}$ \\
\hline Ile & & Aucun & & $\begin{array}{l}\text { Topographie } \\
\text { bifurcation }\end{array}$ & & $\begin{array}{l}\text { Topographie } \\
\text { bifurcation }\end{array}$ \\
\hline Confluence & & $\begin{array}{l}\text { Limites Z.I } \\
\text { confondues }\end{array}$ & & $\begin{array}{l}\text { Concomitance } \\
\text { et effets 2D }\end{array}$ & & $\begin{array}{l}\text { Concomitance } \\
\text { topographie } \\
\text { zone confluence }\end{array}$ \\
\hline $\begin{array}{l}\text { Encaissant } \\
\text { peu marqué }\end{array}$ & & $\begin{array}{l}\text { Limite du lit } \\
\text { majeur erronée }\end{array}$ & & Aucun & & Aucun \\
\hline
\end{tabular}


hydrologique pour la transformation des pluies en débit, ou des traces morphologiques laissées par les crues anciennes (Dezileau et al., 2014). Le projet a permis de mettre au point une méthodologie de comparaison des méthodes probabilistes (Renard et al., 2013) qui pourra servir de référence par la suite. Toute nouvelle méthode ou toute variante de méthode pourra ainsi être évaluée en terme de justesse et de robustesse d'estimation, de façon globale sur un jeu complet de données test et en vérifiant la qualité des résultats sur des sous-ensembles dédiés à une thématique spécifique (taille de bassin versant, régime hydro-climatique). Pour autant, ce protocole n'est approprié que pour des méthodes où une application semi-automatique est possible. On peut considérer que la justesse des méthodes a été correctement évaluée jusqu'à la fréquence de dépassement de $10^{-2}$, du fait de la taille limitée des échantillons (Kochanek et al., 2014). Au-delà, il faudrait raisonner à une échelle régionale, mais en prenant en compte la dépendance spatiale entre séries. La robustesse des modèles a été évaluée jusqu'à la fréquence de dépassement de $10^{-3}$, mais elle n'intervient qu'en second rang dans le choix des méthodes (cf. section III.3).

Trois pistes de développement sont à mentionner :

- L'estimation des crues extrêmes en site non jaugé ou sur un bassin « atypique » (très petit ou très grand bassin versant, influences diverses : karst-neige-zones d'expansion-zone urbaine ...) reste encore très imprécise et doit faire l'objet au cas par cas d'une expertise hydrologique sur les modèles et hypothèses utilisés. Des progrès sont encore nécessaires dans la régionalisation des paramètres des modèles et les bassins atypiques nécessitent des études plus poussées pour prendre en compte leur mode de fonctionnement particulier en crue, et évaluer l'adéquation des méthodes envisagées.

- L'estimation des pluies et crues extrêmes en contexte non stationnaire est possible via le développement en cours de nouveaux modèles aptes à produire une estimation fonction du temps et à intégrer des co-variables climatiques (Tramblay et al., 2012 et 2013 ; Soubeyroux et al., 2014). La position de la France au croisement de plusieurs influences climatiques, l'absence de tendance claire à ce jour sur une évolution généralisée des extrêmes de pluie et de crue en France, et l'insuffisance des modèles climatiques à représenter correctement les valeurs extrêmes font qu'aujourd'hui aucune correction n'est pratiquée par rapport à l'impact du changement climatique. Ce domaine fait l'objet de recherches actives pour le cas échéant faire évoluer les pratiques d'estimation des pluies et crues extrêmes.

- Les modalités d'estimation des incertitudes et de leur prise en compte dans la prise de décision sont largement perfectibles. Le projet a montré l'intérêt de la distribution prédictive pour améliorer notamment l'estimation de lois GEV ou GP calées sur un échantillon local. Il n'en reste pas moins que les incertitudes de modélisation restent encore difficiles à appréhender (Alliau et al., 2014).

L'ensemble des résultats du projet ExtraFlo (Projet ANR-08-RISK-03-01) financé dans le cadre du programme RiskNat de l'Agence Nationale de la Recherche peut être récupéré sur le site http://extraflo.irstea.fr/

\section{REFERENCES}

Alliau D., De Saint Seine J., Lang M., Sauquet E., Renard B. (2014) - Etude du risque d'inondation d'un site industriel par des crues extrêmes : de l'évaluation des valeurs extrêmes aux incertitudes hydrologiques et hydrauliques. La Houille Blanche (à paraître)
ARnAUd P., LAVABRE J. (2000) - La modélisation stochastique des pluies horaires et leur transformation en débits pour la prédétermination des crues. Revue des Sciences de l'Eau. 13(4) : 441-462

Arnaud P., Lavabre J. (2002) - Coupled rainfall model and discharge model for flood frequency estimation. Water Resour. Res. 38 (6) : 11-1-11-11

Arnaud P., LaVABre J. (2012) - Estimation de l'aléa pluvial en France métropolitaine. Eq. Quae. 158 p.

Arnaud P., Eglin Y, Janet B. Et Payrastre O. (2013) - Bases de données SHYREG-débit : Méthode - Performances - Limites. Notice utilisateur. $35 \mathrm{p}$

Arnaud P., Aubert Y., Organde D., Cantet P., Fouchier C., Folton N. (2014) - Estimation de l'aléa hydro-météorologique par une méthode par simulation. La méthode SHYREG : Présentation - Performances - Base de données. La Houille Blanche. 2 : 20-26

Beirlant J., Goegebeur Y., Teugels J.And Segers J. (2004) Statistics of extremes. Theory and applications. Wiley Series in Probability and Statistics. Wiley.

Benito G., Lang M., Barriendos M., Llasat M.C., Frances F., Ouarda T., Thorndycraft V., Enzel Y., Bardossy A., Coeur D., Bobee B. (2004) - Use of Systematic, Palaeoflood and Historical data for the improvement of flood risk estimation. Review of scientific methods. Natural Hazard. Special number "Strategies and Applications in Natural Hazard Research using Historical Data". Edited by T. Glade \& M. Lang, Kluwer Academic Publishers. 31 (3) : 623-643

Beven, K J And Kirkby, M J. (1979) - A physically based variable contributing area model of basin hydrology. Hydrol. Sci. Bull. 24(1) : 43-69

CaYla O. (1993) - Probabilistic calculation of design floods: SPEED. Internat. Symposium on Engineering Hydrology, American Society of Civil Engineers. San Francisco, July.

Cernesson F., Lavabre J., Masson J.M. (1996) - Stochastic model for generating hourly hyetographs. Atmospheric Research. 42(1-4)

Coles S. (2001) - An Introduction to Statistical Modeling of Extreme Values. Springer Series in Statistics, ed. SpringerVerlag. London: Springer-Verlag. 210 p.

Dalrymple T. (1960) - Flood frequency analysis. U.S. Geol. Surv. Water Supply Pap. 1543A

Delgado J.L. (2006) - Estimation des crues de reference par approche géomorphologique. La Houille Blanche. 5 : 97-101

Dezileau L., Terrier B., Berger J.F., Blanchemanche P., Freydier R., Latapie A., Paquier A., Lang M., Delgado J.L. (2014) Reconstitution des crues extrêmes du Gardon à partir d'une analyse paléo-hydrologique. La Houille Blanche (à paraître)

Embrechts P., C. Klüppelberg, And T. Mikosch (1997) Modelling extremal events for insurance and finance. Stochastic modelling and applied probability. Springer. 648

Fisher R.A. AND L.H. TipPETt (1928) - Limiting forms of the frequency distribution of the largest or smallest member of a sample. Cambridge Phil. Soc. 24

Garavaglia F., Gailhard J., Paquet E., Lang M., Garcon R., BERNARDARA P. (2010) - Introducing a rainfall compound distribution model based on weather patterns sub-sampling. Hydrol. Earth Syst. Sci. 14 : 951-964

Garavaglia F., Lang M., Paquet E., Gailhard J., Garcon R., RENARD B. (2011) - Reliability and robustness of rainfall compound distribution model based on weather pattern sub-sampling. Hydrol. Earth Syst. Sci. 15 : 519-532

GARCON R. (1996) - Prévision opérationnelle des apports de la Durance à Serre-Ponçon à l'aide du modèle MORDOR. Bilan de l'année 1994-1995. La Houille Blanche. 5 : 71-76

Garry G., Masson M., Ballais J.L. (1996) - Cartographie des zones inondables : approche hydrogéomorphologique. 
Minist. de l'Equipement, Minist. de l'Environnement, Les Editions Ville et Territoires, Paris La Défense. 100 p.

Gaume E., Gaal L., Viglione A., Szolgay J., Kohnova S., BLoschl G. (2010) - Bayesian MCMC approach to regional flood frequency analyses involving extraordinary flood events on ungauged sites, doi10.1016/j.jhydrol.2010.01.008. J. Hydrol. 394 : 101-117

Guillot P., Duband D. (1967) — La méthode du Gradex pour le calcul de la probabilité des crues à partir des pluies. Colloque International sur les crues et leur évaluation, Leningrad, 15-22 Août, IASH.

Hosking J.R.M. And J.R. Wallis (1997a) - Regional Frequency Analysis, Cambridge University Press. 224 p.

Hosking J.R.M. And J.R. Waldis (1997b) - Regional Frequency Analysis: an approach based on L-Moments. Cambridge, UK: Cambridge University Press. 226 p.

House K.P., WebB R.H., Baker V.R. And Levish D.R. (2002) Ancient Floods, Modern Hazards: Principles and Applications of Paleoflood Hydrology. Water science and Application. 5 AGU

JENKINSON A.F. (1955) - The frequency distribution of the annual maximum (or minimum) values of meteorological elements. Quarterly Journal of the Royal Meteorological Society. 81 : p. 158-171

Kochanek K., Renard B., Arnaud P., Aubert Y., Lang M., Cipriani T., SAuquet E. (2014) — A data-based comparison of flood frequency analysis methods used in France. NHESS 14 : 295-308

Lambert R., Gazelle M., Gholami M., Prunet C. (2001) - La cartographie informative des zones inondables. L'exemple de Midi-Pyrenees. Actes du colloque "Au chevet d'une catastrophe ", Presses Universitaires de Perpignan, Perpignan. p. 147-164

LANG M., LaVABre J. (2007) - Estimation de la crue centennale pour les plans de prévention des risques d'inondations. Editions Quae, Coll. Update Sciences \& Technologies, ISBN 978-2-7592-0067-2, Nov. $232 \mathrm{p}$

Latapie A., Terrier B., Paquier A., Lang M., Chambon D., Doumenc H., Delgado J.L. (2014) - Comparaison des cartographies des zones inondables par approche hydrogéomorphologique et des zones inondées définies par modélisation hydraulique sur la Garonne et les Gardons. La Houille Blanche (à paraître)

Lavabre J., Arnaud P., Masson J.M., Folton N. (1998) - Apport de la modélisation de la pluie en débit pour la connaissance de la ressource en eau et la prédétermination des crues. Congrès de la Société Hydrotechnique de France. 25èmes journées de l'hydraulique, Chambéry, 15-18/09/98. Publications S.H.F. 2 : 349-358

Margoum M. (1992) - Estimation des crues rares et extrêmes : le modèle Agregee. Conceptions et premières validations - Thèse de doctorat, Ecole des Mines de Paris, Cemagref Lyon, GIS Hydrologie Friend-Amhy. 252 p.

Margoum M., Oberlin G., Lang M., Weingartner R. (1994) Estimation des crues rares et extrêmes : principes du modèle Agregee. Hydrologie Continentale. 9(1) : 85-100

Miquel J. (1984) - Guide pratique d'estimation des probabilités de crues. Ed. Eyrolles. 160 p.

Neppel L., Renard B., Lang M., Ayral P.A., Coeur D., Gaume E., Jacob N., Payrastre O., Pobanz K., Vinet F. (2010) — Flood frequency analysis using historical data: accounting for random and systematic errors. Hydrological Sci. J. 55(2) : 192-208

Onibon H., Ouarda T.B.M.J., Barbet M., St-Hilaire A., Bobee B., Bruneau P. (2004) - Analyse fréquentielle régionale des précipitations journalières maximales annuelles au Québec. Hydrol. Sci. J. 49 (4) : 717-735

PAQUeT E. (2004) - Evolution du modèle hydrologique MORDOR : modélisation du stock nival à différentes altitudes. La Houille Blanche. 2 : 75-82
Paquet E., Gailhard J., Garçon. R. (2006) — Evolution de la méthode du Gradex : Approche par type de temps et modélisation hydrologique. La Houille Blanche. 5 : p. 80-90

Paquet E., Lang M., Carre J.C. (2012) - SCHADEX method for extreme flood estimation: overview, applications and perspectives. 24th ICOLD Congress - Q94. ICOLD-CIGB, Kyoto.

Paquet E., Garavaglia F., Garçon R., Gailhard J. (2013) — The SCHADEX method: a semi-continuous rainfall-runoff simulation for extreme flood estimation. J. Hydrol. 495 : 23-27

Paquet E., Aubert Y., Arnaud P., Royet P., Fine J.A. (2014) Prédétermination des crues et cotes de projet au moyen des méthodes SHYPRE et SCHADEX. Application à un aménagement fictif sur le Tech. La Houille Blanche (à paraître)

PARent E., BERnier J. (2003) - Bayesian POT modelling for historical data. J. Hydrol. 274 : 95-108

Payrastre O., Gaume E., Andrieu H. (2011) — Usefulness of historical information for flood frequency analyses: developments based on a case study. Water Resour. Res. 47 : W08511

PERRIN C. (2000) — Vers une amélioration d'un modèle global pluie-débit au travers d'une approche comparative. Thèse de Doctorat, INP Grenoble, Cemagref Antony. 530 pp.

PICKANDS J. (1975) - Statistical inference using extreme order statistics. Annals of Statistics. 3 : 119-131

PRunet C. (1998) - Géographie des zones inondables de la Dordogne Moyenne - Thèse doctorat de Géographie Aménagement Université Toulouse le Mirail.

Renard B., Kochanek K., Lang M., Garavaglia F., Paquet E., Neppel L., Najib K., Carreau J., Arnaud P., Aubert Y., Borchi F., Soubeyroux J.M., Jourdain S., Veysseire J.M., Sauquet E., Cipriani T., Auffray A. (2013) - Data-based comparison of frequency analysis methods: A general framework. Water Resour. Res. 49 : 1-19

Ribatet M., Sauquet E., Gresillon J.M., Ouarda T.B.M.J. (2006) - A regional Bayesian POT model for flood frequency analysis. Stochastic Env. Res. Risk A. 21(4) : 327-339

Ribatet M., Sauquet E., Gresillon J.M., Ouarda T.B.M.J. (2007) - Usefulness of the reversible jump Markov chain Monte Carlo model in regional flood frequency analysis. Water Resour. Res. 43(8)

Sheffer N. A., Enzel Y., Benito G., Grodek T., PoArt N., LAng M., Naulet R., Coeur D. (2003a) - Paleofloods and historical floods of the Ardèche river, France. Water Resour. Res. 39 (12), $1376: 13 \mathrm{p}$.

Sheffer N. A., Enzel Y., Waldmann N., Grodek T. (2003b) Claim on largest flood on record proves false. EOS Transactions, American Geophysical session, 12, 25 March 2003. 84

St-Hilaire A., Ouarda T.B.M.J., Lachance M., Bobee B., Barbet M., BRUNEAU P. (2003) - La régionalisation des précipitations: une revue bibliographique des développements récents. Rev Sci. Eau. 16 (1) : 27-54

Soubeyroux J.M., Neppel L., Veysseire J.M., Gouget V. (2014) - Evolution des précipitations extrêmes en France en contexte de changement climatique. La Houille Blanche (à paraître)

Tramblay Y., Neppel L., Carreau J., Sanchez-Gomez E. (2012) - Extreme value modelling of daily areal rainfall over Mediterranean catchments in a changing climate. Hydrol. Process. 26 : 3934-3944

Tramblay Y., Neppel L., Carreau J., Najib K. (2013) Non-stationary frequency analysis of heavy rainfall events in Southern France. Hydrological Sciences Journal. 58(2) : 280-294

VEN Te CHOw (1964) - Handbook of applied hydrology. Hydrology of Agricultural Lands - MacGraw-Hill - 1964. 20

VIDAL JJ (2000) - Cartographie informative des zones inondables en Midi-Pyrénées. Une démarche à l'échelle de la région. Colloque national sur les risques naturels, au Cemagref de Grenoble, 28 et 29 septembre 2000. 